\title{
Muscle Imbalance in Volleyball Players Initiates Scoliosis in Immature Spines: A Screening Analysis
}

\author{
Hitesh Modi*, S Srinivasalu*, Satyen SMehta*, Jae-Hyuk Yang ${ }^{\dagger}$, \\ Hae-Ryong Song*, Seung Woo Suh* \\ *Scoliosis Reseach Institute, Korea University Guro Hospital, Seoul, Korea, \\ ${ }^{\dagger}$ Rare Disease Institute, Korea University Guro Hospital, Seoul, Korea
}

Study Design: Retrospective comparative study using radiographs and clinical findings.

Purpose: To test the hypothesis that asymmetric loading of immature spines in young athletes initiates scoliosis.

Overview of Literature: Scoliosis in athletes has been reported in the literature, but its causative factors have not been investigated.

Methods: We compared the incidence, type and magnitude of scoliotic curves in volleyball players with those in the nonplayer population. One hundred sixteen adolescent volleyball players were grouped for selective screening. Data regarding their playing duration, handedness, age, height, and menarchal status (in girls) were recorded, along with clinical examination and radiological investigation when necessary. We analyzed data from 46,428 non-player school children, and their data were compared to athletes to determine differences.

Results: Volleyball players had a statistically significant increase in the incidence of scoliotic spinal curves. Playing hand dominance was related to the curve direction. Cobb angle had no significant correlation with the duration of playing.

Conclusions: There is a five-fold increase in the incidence of mild scoliosis in volleyball players. A high percentage (41\%) of asymmetry was present on the Adams forward bending test, as compared to controls. The curves were either thoracic or thoracolumbar.

Key Words: Volleyball players, Asymmetric loading, Column buckling, Scoliosis, Etiology

\section{Introduction}

The etiology of adolescent idiopathic scoliosis (AIS) is still not completely understood. Many theories have been proposed in the literature. Proposed etiologies include mechanical ${ }^{1,2}$, hormonal ${ }^{3,4}$, muscular $^{5,6}$, biochemical $^{7,8}$, genet$\mathrm{ic}^{9,10}$, neurologic ${ }^{11,12}$, and other causes. Currently, multi-factorial etiology is the most accepted cause. The Hueter-Volkmann theory for the development of deformity in the spine demonstrates that compression or loading at end plates retards growth, and distraction accelerates growth in the end plates of vertebrae in the immature spine. Wedging of verte- bral bodies is part of the etiology for the development of scoliosis in the immature spine. Tanchev et al..$^{13}$, suggested that asymmetric muscle force, along with ligament laxity and delayed menarche, might disturb the balancing mechanism of the spine and could possibly initiate the development of scoliosis. Scoliosis appears during the growth spurt ${ }^{14}$, leading initially to cosmetic deformities. With progression, scoliosis can lead to various disabilities and limitations $\mathbf{s}^{15}$.

Scoliosis has been reported to occur with increased frequency in ballet dancers ${ }^{16}$ and in rhythmic gymnasts ${ }^{13}$. The increased incidence of scoliosis associated with these professions is attributed to persistent asymmetric loading of the

Corresponding author: Seung Woo Suh, MD

Department of Orthopedics, Korea University Guro Hospital, Korea University College of Medicine

\#80, Guro-dong, Guro-gu, Seoul, Korea

Tel: +82-2-2626-3087, Fax: +82-2-867-1145, E-mail: spine@korea.ac.kr 
growing spine secondary to disturbed balancing of muscles, along with ligament laxity and delayed menarche. Harrington $^{17}$ postulated that the deformed state of the growing spine is a reaction of structural living matter to abnormal physical forces. The literature suggests that patients with idiopathic scoliosis have a tendency to be taller than their peers. This pattern has been implicated in column buckling ${ }^{18,19}$.

We postulated that repeated asymmetric rotational and bending strain along with column buckling in the spines of volleyball players produce an imbalance in the stabilizing mechanism of the muscles, leading to scoliosis. We conducted a selective study in middle school and high school volleyball players and compared them with a control group of non-volleyball players (no sports at all). We compared the incidence, type, and magnitude of scoliotic curves and the relationship between handedness and curve incidence in both groups. We also correlated the curve severity with the length of play. We then tried to determine the etiology for initiation of scoliosis in athletes.

\section{Materials and Methods}

\section{Study group}

One hundred sixteen middle school and high school volleyball players with an average age 15.2 years (range, 11.1 to 18.9 years) were chosen for selective screening ${ }^{20}$ from five centers in and around Seoul, Korea. After informed consent was obtained, a clinical examination of the back was undertaken. We performed a gross inspection, Adams forward bending test, and angle of trunk rotation (ATR) test using a scoliometer, as described by Bunnel ${ }^{21}$ (Scoliometer made by Orthopedic systems Inc, USA). We further investigated those players who had an ATR of $5^{\circ}$ or more. We excluded children who had signs of neurological involvement or ligament laxity, or who had a previous history of spine injury or any kind of neurological disorder such as spina bifida or neurofibromatosis. We also excluded those children who had congenital scoliosis or juvenile scoliosis on examination. Players were also examined clinically to rule out any limb length discrepancy that might cause functional scoliosis ${ }^{22}$ and affect the study outcome. For female patients, we determined menstrual history, onset of menarche, and any irregularities, if present. Standing height and handedness were also recorded. Any family history of spinal deformity or back pain, or previous treatment for the same, were also assessed.

We noted the average number of hours training or playing each week and the total duration of play for all athletes. We excluded players who had played or trained for less than eighteen months. We also inquired about involvement in other games or sports. If a curve was detected on clinical examination with the Adams forward bending test, the player was subjected to standing postero-anterior (PA) long radiographs and side bending films of the spine. The curves were then classified ${ }^{22}$, and the Cobb angle was calculated according to the Cobb-Lipmann technique ${ }^{23}$. The apical vertebral rotation was calculated using the method of Nash and $\mathrm{Moe}^{24}$. All results were recorded for analysis and compared with the control group.

\section{Control group}

We also studied 46,428 middle school and high school children from forty-five schools (age range, 11 to 15 years), who had been examined as part of a school screening program in Seoul. All these children were enrolled as a control group. There were 24,892 boys and 21,536 girls (ratio, $\mathrm{M}: \mathrm{F}=1: 0.86)$. We obtained data related to the number of curves, curve characteristics, Cobb angle, ATR, and other tests. Students who had already been diagnosed and were being treated with braces, those who had undergone surgery, and those who had refused surgery, were also included in the control group. Normative data for Korean children with regard to average height were sourced from the University of Minnesota international adoption clinic growth chart for Korean boys and girls. The average age of menarche was $12.5 \pm 2$ years for Korean girls ${ }^{25,26}$.

\section{Analysis}

The prevalence rate of scoliosis and ATR in players and control subjects was compared statistically using Fischer's exact test. The relationships between handedness and scoliosis, menarchal status and scoliosis, and vertebral rotation and scoliosis were also determined to highlight any relationship between them. The results of the present study were compared with the school screening study for relevance and strength of association.

\section{Results}




\section{Volleyball players}

In this study, the male:female ratio was 0.84:1 (54 boys and 62 girls). The average period of intense playing or training for volleyball was 4.2 years (range, 1.6 to 8.2 years). Players were predominantly right-handed in the game, as well as in their activities of daily living (109 out of 116). There were no players with differing hand dominance for playing and for daily living. There were no major spine injuries or players under treatment for back pain. Sixtythree percent (74 out of 116) of the players were taller than the 97th percentile for age. There were no female players with delayed menarche or with amenorrhea.

\section{Scoliosis curve}

Six curves were greater than ten degrees, recognized on radiograms, with ATR greater than five degrees (20 players). The average Cobb angle for scoliosis players was $13^{\circ}$ (range, 11 to $17^{\circ}$ ), and the average ATR for scoliosis players was $8^{\circ}$ (range, 7 to $11^{\circ}$ ). There were five curves with convexity to the right and one with convexity to the left. All six curves had their apex at the lower thoracic (three players) or thoracolumbar (three players) level. There were no high thoracic, lumbar, or double major curves. Comparison of the number of curves and the Cobb angle of the curves in players versus the control group is depicted in Table 1.

There was a higher prevalence of scoliosis in the study group ( $\mathrm{p}=0.000007)$ than in the control group: $5.2 \%$ versus $1 \%$, respectively. Even though the control group had a predominance of single thoracic $(26.7 \%)$ or double thoracic and lumbar curves (35.3\%), thoracolumbar (15.3\%), double thoracic $(1.7 \%)$, and lumbar curves $(21.1 \%)$ were also iden- tified. The curve magnitude and the duration of play were not correlated $\left(\mathrm{r}^{2}=0.0014\right)$.

\section{Angle of trunk rotation}

Truncal asymmetry by Adams forward bending test was positive in 48 of 116 players (41\%). Greater than five degrees of ATR was identified in 20 players (12 right and 8 left). The ATRs for both groups are compared in Table 2S. The duration of play and the ATR showed no statistical relationship $\left(r^{2}=0.004\right)$.

\section{Discussion}

We tried to determine the role of asymmetric back exercise in the initiation of scoliosis curves. We selected young volleyball players for the study, as the game involves repetitive twisting and jumping, with the back arched during spiking, jump serving, and blocking. The main load is transferred through the upper limbs to the clavicles, and then to the thoracic spine. This results in a very high rotational and mostly unilateral strain on the back. Moreover, volleyball is a predominantly one-handed game, which leads to hypertrophy of muscles on the dominant side, causing an imbalance in muscle activity. An increased prevalence of scoliosis has been reported in ballet dancers ${ }^{16}$, rhythmic gymnasts ${ }^{13}$, swimmers $^{27}$, and skiers $^{28}$. A statistically significant increase in the prevalence among volleyball players may point to repetitive asymmetric muscular contraction in the initiation of scoliotic curves. Arkin ${ }^{29}$ has suggested that mild or functional curves may initiate the structural curve in some cases.

A scoliosis-screening test is not intended to be diagnos-

Table 1. Comparison of the Cobb's angle between the volleyball players and control

\begin{tabular}{lccccc}
\hline Group & Cobb's angle $>10^{\circ}$ & Total number & Percent $\%$ & Average degrees & Range degrees \\
\hline Volleyball player & 6 & 116 & 5.2 & 12 & $10 \sim 15$ \\
Control & 465 & 46,428 & 1.0 & 24.5 & $10 \sim 55$ \\
\hline
\end{tabular}

Fishers exact test: $\mathrm{p}=0.000007$.

Odds ratio, $6.1 ; 95 \% \mathrm{CI}, 3.2$ to 12.1 .

Table 2. Comparison of the Angle of Truncal Rotation (ATR)

\begin{tabular}{lrrccc}
\hline Group & ATR $>5^{\circ}$ & Total & Percent $\%$ & Average degrees & Range degrees \\
\hline Volleyball players & 20 & 116 & 17 & 7.1 & $0 \sim 9$ \\
Control & 1,155 & 46,428 & 2.5 & 6 & $0 \sim 24$ \\
\hline
\end{tabular}

Fischer exact test p-value $<0.0001$. 
$\mathrm{tic}^{21}$. It is intended only to identify at-risk individuals, and as such, it has more false positives than false negatives. In most such programs, anyone with both trunk asymmetry on the Adams test and positive rotation on scoliometer greater than five degrees is considered positive. The prevalence of scoliotic curves greater than ten degrees in our control group was $1 \%$ ( 465 out of 46,428 ). Such a low prevalence suggests that screening of high-risk groups is more effective $^{20}$. In our study, 48 players (41\%) had some degree of truncal asymmetry on the Adams forward bending test. Even though small amounts of truncal asymmetry are reported to be common in school children without scoliosis $^{15,30}$, ATR more than five degrees was present in twenty players $(17 \%)$ in our study. We fixed the limit of referral at five degrees, for when five degrees of rotation is used, the number of twenty-degree curves missed on exam is only about $2 \%{ }^{31}$.

In our study group, the average duration of training was $25 \pm 5$ hours per week for an average period of 4.2 years (range, 1 to 8.2 years). These player-students spent the second half of their day in training and were encouraged to continue with the same game for longer periods. The players were generally taller and heavier than their peers, probably because of physical activity and the preference for taller people in volleyball. Some authors have suggested that children with idiopathic scoliosis have a tendency to be taller than their peers, and height has been implicated in the tendency towards column buckling ${ }^{18,19}$. Delayed puberty is a known risk factor for scoliosis ${ }^{16}$. We did not note a significant delay in menarche or menstrual irregularity warranting a gynecologic exam in any of our players.

Scoliotic curves greater than ten degrees were identified in the radiograms of six players (5.2\%), and all of these players also had ATRs greater than five degrees. Interestingly, we found all scoliosis players had ATRs of at least $7^{\circ}$, which was also suggested by Bunnel ${ }^{31}$. Bunnel proposed the criteria for scoliosis screening using a scoliometer and also suggested that the ATR referral criteria should be $7^{\circ}$. In this study, the volleyball players had a five-fold increase of scoliosis prevalence compared to the general student population (1\%). Scoliosis as measured by Cobb's angle does not always correlate with asymmetry or rib rotation ${ }^{32}$. This may explain the low proportion of curves and very high prevalence of trunk asymmetry in the player group. About $17 \%$ of players (20 players) had significant truncal asymmetry. This discrepancy in incidence of truncal asymmetry and real scoliosis may be due to asymmetric paraspinal muscle volumes in athletes. Incidentally, the natural history committee of the British Scoliosis Society ${ }^{33}$ (BSS) has stated that the mean ATR in normal boys is $0.6 \pm$ 2.6 degrees and in girls is $0.8 \pm 2.7$ degrees. Our study showed a twenty-fold increase of greater than five degrees of ATR in volleyball players.

On radiological examination, the apical vertebrae in these curves were located in the lower thoracic or the thoracolumbar level (Fig. 1). This localization in the thoracolumbar area seems to be principally the result of repetitive asymmetric stresses in the transitional region of the spine. As the thoracolumbar spine is between the rigid thoracic spine and the more mobile lumbar spine, a high proportion of stress is concentrated in this area during volleyball play. This is in concurrence with Warren et al. ${ }^{16}$, who have reported mainly mild thoracic curves in ballet dancers. Long-term follow-up of players who continue with the game is required to assess the course of spinal curves.

Asymmetric muscle exercise and overloading, along with

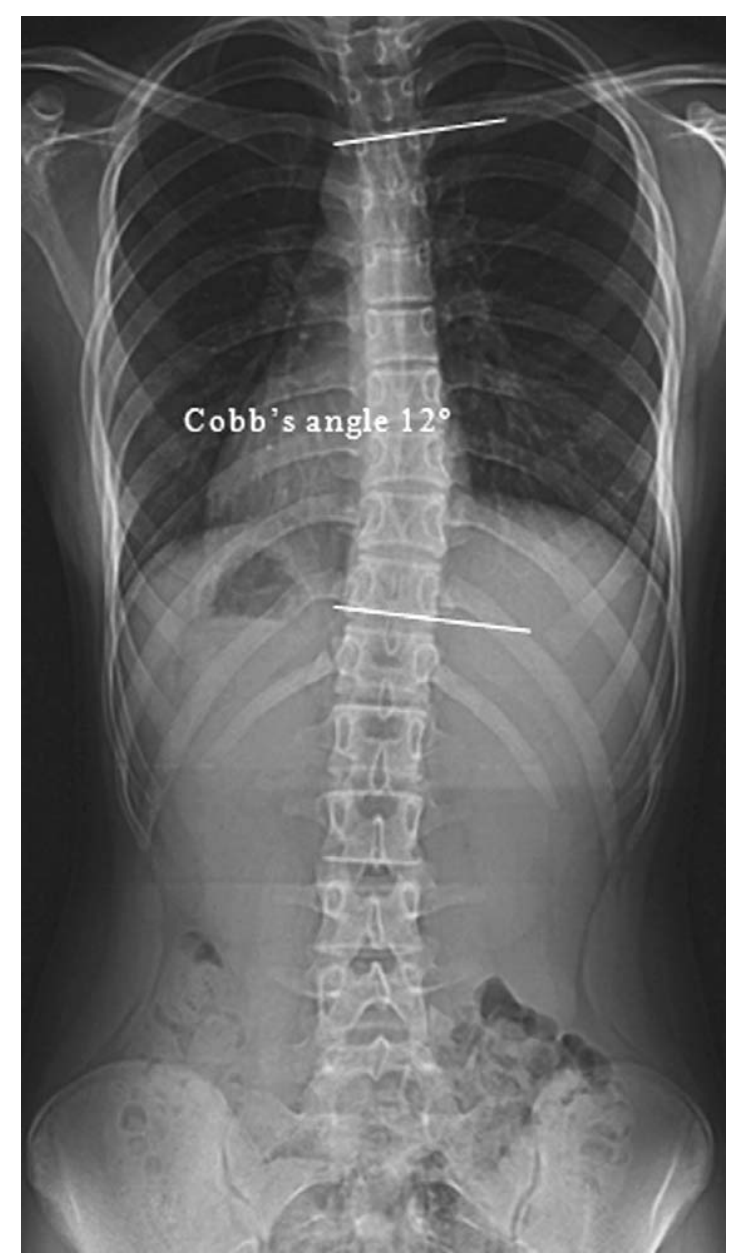

Fig. 1. Postero-anterior $x$-ray of the whole spine showing a mild lower thoracic curve in a volleyball player. 
column buckling due to increased height compared with peers, are certainly initiating factors for these mild curves, as can be inferred from the five-fold increase in curve prevalence among players. Tanchev et al..$^{13}$, suggested this, as well. Asymmetry in muscle force may also disturb the balancing mechanism of the spine ${ }^{34}$. The Hueter-Volkmann law is applicable in these situations where extended periods of asymmetric twisting and loading in the backs of volleyball players can lead to deformations in the body. However, we could not identify any vertebral wedging in the radiographs of the players. Schultz and Galante ${ }^{35}$ have shown that mild scoliotic curves can be produced in apparently normal spines with no changes in the vertebral bodies.

In this study, 93\% (109 out of 116) of players were righthanded, and five of them had scoliotic curves greater than ten degrees to the right. Of the seven left-handed players, only one developed a scoliotic curve to the left. Handedness has been shown to be significantly related to the direction of the primary curve. It has also been shown to be related to the direction of the lower thoracic curve, regardless of the primary or structural curve ${ }^{36}$. There were not enough positive results in our study to support or refute this association. Neither the age at which training started nor the duration of play showed any correlation with the magnitude of the scoliotic curve. A future study with long-term follow-up of a large number of very young players needs to be conducted to establish whether repetitive asymmetric loading or column buckling due to height leads to structural scoliosis.

Sports-associated scoliosis has been put forward as a separate diagnostic entity ${ }^{13}$, and we support the case for this distinct subgroup under the classification of scoliosis. We also suggest that sports involving predominantly upper limb exercise create an imbalance in the weight-transferring mechanism on the spine, which results in initiation of scoliosis curving along with column buckling. There is a tendency toward rapid growth early in adolescence, just when scoliosis is most prone to increase.

\section{Conclusions}

These young volleyball players had a five-fold increase in the incidence of curves, but they were mild between ten and fifteen degrees and were located in the lower thoracic or thoracolumbar spine. There was a nearly twenty-fold increase in the incidence of significant truncal asymmetry in players compared with controls. The curve pattern matched handedness in our study, but the duration of play did not correlate with the onset of scoliosis. Long-term studies with larger numbers of patients are needed to determine if progression of functional scoliosis to structural scoliosis is induced by asymmetric spine twisting, loading, and column buckling due to increased height. Furthermore, sports-associated scoliosis should be considered a distinct subgroup of scoliosis.

\section{REFERENCES}

1. Andriacchi T, Schultz A, Belytschko T, Galante J: A model for studies for mechanical interactions between the human spine and rib cage. J Biomech 1974; 7: 497-507.

2. Arkin AM: The mechanism of rotation in combination with lateral deviation in the normal spine. J Bone Joint Surg Am 1950; 32: 180-188.

3. Machida M, Dubousset J, Imamura Y, Iwaya T, Yamada T, Kimura J: An experimental study in chickens for the pathogenesis of idiopathic scoliosis. Spine 1993; 18: 1609-1615.

4. Mente PL, Stokes IA, Spence H, Aronsson DD: Progression of vertebral wedging in an asymmetrically loaded rat tail model. Spine 1997; 22: 1292-1296.

5. Riddle HF, Roaf R: Muscle imbalance in the causation of scoliosis. Lancet 1955; 18: 1245-1247.

6. Stokes IA: Analysis of symmetry of vertebral body loading consequent to lateral spinal curvature. Spine 1977; 22: 2495-2503.

7. Bushell GR, Ghosh P, Taylor TK, Sutherland JM: The collagen of the intervertebral disc in adolescent idiopathic scoliosis. J Bone Joint Surg Br 1979; 61: 501-508.

8. Ponseti IV, Pedrini V, Dohrman S: Biochemical analysis of intervertebral discs in idiopathic scoliosis. J Bone Joint Surg Am 1972; 54: 1793.

9. Cowell HR, Hall JN, MacEwen GD: Genetic aspects of idiopathic scoliosis. A Nicholas Andry Award essay, 1970. Clin Orthop Relat Res 1972; 86: 121-131.

10. Wynne-Davies R: Familial (idiopathic) scoliosis. A family survey. J Bone Joint Surg Br 1968; 50: 24-30.

11. Herman R, Mixon J, Fisher A, Maulucci R, Stuyck J: Idiopathic scoliosis and the central nervous system: A motor control problem. The Harrington lecture, 1983. Scoliosis Research Society. Spine 1985; 10: 1-14.

12. Yekutiel M, Robin GC, Yarom R: Proprioceptive func- 
tion in children with adolescent idiopathic scoliosis. Spine 1981; 6: 560-566.

13. Tanchev PI, Dzherov AD, Parushev AD, Dikov DM, Todorov MB: Scoliosis in rhythmic gymnasts. Spine 2000; 25: 1367-1372.

14. Risser JC: The iliac apophysis; an invaluable sign in the management of scoliosis. Clin Orthop 1958; 11: 111-119.

15. Vercauteren M, Van Benden M, Verplaetse R, Croene P, Uyttendaele D, Verdonk R: Trunk asymmetries in a Belgian school population. Spine 1982; 7: 555-562.

16. Warren MP, Brooks-Gunn J, Hamilton LH, Warren LF, Hamilton WG: Scoliosis and fractures in young ballet dancers. Relation to delayed menarche and secondary amenorrhea. N Eng J Med 1986; 314: 1348-1353.

17. Harrington PR: The etiology of idiopathic scoliosis. Clin Orthop Relat Res 1977; 126: 17-25.

18. Shohat M, Shohat T, Nitzan M, Mimouni M, Kedem R, Danon YL: Growth and ethnicity in scoliosis. Acta Orthop Scand 1988; 59: 310-313.

19. Carr AJ, Jefferson RJ, Turner-Smith AR: Family stature in idiopathic scoliosis. Spine 1993; 18: 20-23.

20. Bunnell WP: Selective screening of scoliosis. Clin Orthop Relat Res 2005; 434: 40-45.

21. Bunnell WP: An objective criterion for scoliosis screening. J Bone Joint Surg Am 1984; 66: 1381-1387.

22. Lonstein JE: Idiopathic scoliosis (in Lonstein JE, Winter RB, Bradford DS, Ogilvie JW eds. Moe's textbook of scoliosis and other spinal deformities. 3rd ed. Philadelphia, WB Saunders Co: 219, 1995).

23. Cobb JR: Outline for the study of scoliosis. American Academy of Orthopaedic Surgeons Instr Course Lect 1948; 5: 261-275.

24. Nash C, Moe J: A Study of Vertebral Rotation. J Bone J Surg 1969; 51: 223-229.
25. Kim KH, Spurgeon JH, French KE, Kim KB: Somatic comparisons of South Korean children and youths born and reared in a rural area with the descendants of rural to urban migrants. Am J Hum Biol 2002; 14: 476-485.

26. Park SH, Shim YK, Kim HS, Eun BL: Age and seasonal distribution of menarche in Korean girls. J Adolesc Health 1999; 25: 97.

27. Becker TJ: Scoliosis in swimmers. Clin in Sports Med 1986; 5: 149-158.

28. Ogon M, Riedl-Huter C, Sterizinger W, Krismer M, Spratt KF, Wimmer C: Radiologic abnormalities and low back pain in elite skiers. Clin Orthop Relat Res 2001; 390: 151-162.

29. Arkin AM: The mechanism of the structural changes in scoliosis. J Bone Joint Surg Am 1949; 31: 519-528.

30. Burwell RG, James NJ, Johnson F, Webb JK, Wilson YG: Standardized trunk asymmetry scores. A study of back contour in healthy school children. J Bone Joint Surg Br 1983; 65: 452-463.

31. Bunnell WP: Outcome of spinal screening. Spine 1993; 18: $1572-1580$

32. Armstrong GW, Livermore NB 3rd, Suzuki N, Armstrong JG: Nonstandard vertebral rotation in scoliosis screening patients. Its prevalence and relation to the clinical deformity. Spine 1982; 7: 50-54.

33. Burwell G: The British decision and subsequent events. Spine 1988; 13: 1192-1194.

34. Pal GP: Mechanism of production of scoliosis. A hypothesis. Spine 1991; 16: 288-292.

35. Schultz AB, Galante JO: A mathematical model for the study of the mechanics of the human vertebral column. J Biomech 1972; 3: 405-416.

36. Goldberg C, Dowling FE: Handedness and scoliosis convexity: a reappraisal. Spine 1990; 15: 61-64. 